\title{
End-diastolic and End-systolic LV morphology in the presence of cardiovascular risk factors: A UK Biobank study
}

\author{
Kathleen Gilbert ${ }^{1}$, Avan Suinesiaputra ${ }^{2}$, Stefan Neubauer, Stefan Piechnik, Nay Aung \\ Steffen E. Petersen ${ }^{3}$, Alistair Young ${ }^{4}$ \\ ${ }^{1}$ Auckland Bioengineering Institute, University of Auckland, New Zealand \\ ${ }^{2}$ Department of Anatomy and Medical Imaging, University of Auckland, New Zealand \\ ${ }^{3}$ William Harvey Research Institute, NIHR Barts Biomedical Research Centre, Queen Mary \\ University of London \\ ${ }^{4}$ Department of Biomedical Engineering, King's College London, London, UK \\ kat.gilbert@auckland.ac.nz
}

\begin{abstract}
Left ventricular function and morphology have been shown to be important factors in clinical and pre-clinical cardiovascular disease. In this paper we used atlas-based techniques to capture the full extent of morphological changes at end-diastole, end-systole and in a coupled functional atlas for 4547 UK Biobank participants. The morphological differences between participants with risk factors for cardiovascular disease were tested using a logistic regression model. The result was compared to a model built from traditional mass and volume measures, and the strength of associations were tested using a Delong's test. Atlas based models had stronger associations with risk factors than mass and volume parameters in most risk factors. The functional atlas showed better performance than the separate end-diastole and end-systole atlases.
\end{abstract}

Keywords: Cardiac MRI, Cardiac Atlases, Cardiovascular Risk Factors

\section{Introduction}

Cardiovascular disease is the leading cause of death worldwide, and is responsible for one in every four deaths [1]. Left ventricular (LV) function and morphology are important predictors of cardiac health. The LV changes shape, in a process known as remodeling, in response to a variety of clinical and pre-clinical disease processes [2]. Previous work has demonstrated changes to LV morphology in those with higher blood pressure [3], higher fat mass [4] and smoking [5]. Many measures of changes to morphology ignore large amounts of information, which is available in cardiac MRI. Statistical shape atlases capture the variety of LV morphological differences [6]. Atlases of the left ventricle have been used to quantify shape differences in participants of longitudinal studies [5,7]. While some previous studies have defined shape measures end- 
diastole (ED), e.g mass to volume ratio and sphericity, others are defined at end-systole (ES), e.g. ES volume index.

UK Biobank is a longitudinal cohort study in the United Kingdom, which has performed cardiac MRI on participants to examine the pre-clinical determinants of cardiovascular disease. The cohort contains a subset of healthy participants who have no cardiovascular risk factors.

Here, we extend previous work which showed that different shape atlases perform similarly in quantifying relationships with cardiovascular risk factors in UK Biobank, and are robust to different methods used in their construction. That work combined the scores from the first 20 modes of the separate ED and ES statistical shape models to quantify the relationships with risk factors. Here, we provide the following novel contributions:

1. We show that separate ED and ES statistical shape models have similar discrimination power for the risk factors in UK Biobank, with ES having higher AUC, suggesting that LV morphology at ES may be more sensitive to the presence of risk factors.

2. We test a functional atlas constructed by concatenating ED and ES shape models, thereby forming a coupled atlas which simultaneously describes coupled ED and ES shape variations. The functional atlas outperformed the separate ED and ES atlases for the diabetes and high cholesterol.

\section{Methods}

\subsection{Imaging protocol and analysis}

The CMR protocol has been descripted previously in [8]. Briefly, all images were acquired on a Siemens AERA 1.5T wide-BORE MRI scanner (Siemens Healthineers, Erlangen, Germany) using retrospectively gated cine balanced steady-state free precession breath-hold acquisitions. A short axis stack covering the left and right ventricles, as well as horizontal and vertical long axis slices and the left ventricular outflow track were acquired. The short axis images were manually contoured at ED and ES as reported in [9]. All contouring was completed in accordance with Society of Cardiovascular Magnetic Resonance recommendations as described in [9].

\subsection{Atlas creation}

The atlas was constructed using the method described in [5]. Briefly, the manual contours were extracted and fitted to a finite element model of the left ventricle using a least squares minimization. The model was orientated using landmarks from the manual contours to denote the LV apex, mitral valve inserts and right ventricle. The orientated model was then fitted to the manual contours at ED and ES, creating participant specific models of the left ventricle.

The participant specific models were then used to create three atlases (ED, ES and a functional atlas). For the individual ED and ES atlases each model was first aligned to 
the average model using Procrustes (rotation and translation only). The coupled functional atlas (EDES atlas) aligned at both ED and ES using the Procrustes calculated for the ED model. Principal component analysis (PCA) was then used to create each atlas. The ED and ES atlas describe shape at each individual time point. The functional atlas (EDES atlas) was created from participants coupled shapes where the surfaces were concatenated at ED and ES during the PCA, thus describes the variations in ED and ES that occur together.

\subsection{Statistics}

Z-scores were extracted for each participant in each mode of each of the three atlases. Associations between cardiovascular risk factors and shape were examined using a fivefold cross-validated logistic regression model in the $\mathrm{R}$ package Caret ( $\mathrm{R}$ version 3.5.1 and Caret version 6.0-81). A separate model was created for each risk factor, which compared the healthy risk-free cohort and those with the particular risk factor. Participants with angina, and those who reported having had a heart attack were excluded from the analysis, except in the case of angina as a risk factor where only those who reported having a heart attack were excluded. Separate models were created for each atlas, as well as a mass and volume model. Area under the curve was recorded. Models were tested using the first 50 modes of variation from each atlas, and the final number of modes was selected where the area under the curve stabilized. Differences between the result of the mass volume model and the atlas models were tested using a Delong's test and a Bonferroni corrected p-value of 0.0125 was considered significant. Risk factors of self-reported hypertension, high cholesterol, angina, smoking, diabetes as well as those who reported taking blood pressure medication or cholesterol medication at the time of imaging were selected for analysis.

\subsection{Visualization of the statistical models}

Let $X \in R^{N \times P}$ be the centered data shape matrix with $N$ cases and $P$ shape dimensions. Principal component analysis of $X$ can be estimated by singular value decomposition of covariance of $X$, i.e. $C=X^{T} X /(N-1)$, as follows

$$
C=\Phi L \Phi^{T}
$$

where $\Phi \in R^{P \times W}$ is an orthonormal matrix of the principal components, $L \in R^{P \times P}$ is a diagonal matrix of eigenvalues (variances of each mode), and $W \leq P$ is the number of principal components or modes of variation. Let $x$ represent an individual column shape vector of $X$ with $P$ shape dimensions. The normalized PCA z-scores for each participant in each mode can be defined as:

$$
T_{z}=x \Phi \sqrt{L}^{-1}
$$

The statistical model defines a regression score as:

$$
Y=T_{z} \beta=T \sqrt{L}^{-1} \beta
$$


Where $Y$ is the score (log-odds in the logistic regression), $\beta \in R^{1 \times Q}$ are the coefficients of the statistical model where $Q$ is the number of variables included in the regression model. We can define the following morphometric scores:

$$
Y_{1}=T \beta_{1}=X \Phi \beta_{1}=X m
$$

Where $\beta_{1}$ is the normalized scaled coefficients $\left(\sqrt{L}^{-1} \beta /\left|\sqrt{L}^{-1} \beta\right|\right)$, and $m$ is a unit length morphometric shape vector. Thus we can visualize shape from the statistical model as

$$
x_{M}=x m m^{T}=Y_{1} m^{T}
$$

The resulting logistic regression models were evaluated for all participants included in the model and visualized at the $5^{\text {th }}$ and $95^{\text {th }}$ percentiles.

\section{Results}

Table 1 shows the LV volumes and mass of participants with each risk factor, and the risk free cohort, as well the number in each group. Figure1 shows the first five PCA modes in the independent ED and ES atlases. The atlases were visually inspected to categorize mode shapes. Modes 1 of both atlases show that size accounts for $46 \%$ of the variation in LV morphology at ED and $43 \%$ at ES. Mode 2 shows variations in sphericity of the ventricle accounting for $15 \%$ and $10 \%$ at ED and ES respectively. The ED atlas shows variation in the mitral valve tilt in modes 3,4 and apical sphericity in mode 5. The ES atlas shows variation in global wall thickness in mode 3 and mitral valve tilt in modes 4 and 5 .

Table 1. Cardiovascular function and number of participants in each risk factor or cohort in-

\begin{tabular}{|c|c|c|c|c|c|}
\hline & $\mathrm{n}$ & Male & $\begin{array}{cc}\text { LV } & \text { EDV } \\
(\mathrm{ml}) & \end{array}$ & LV ESV(ml) & LV mass (g) \\
\hline $\begin{array}{l}\text { Risk free co- } \\
\text { hort }\end{array}$ & 751 & 344 & $143.74 \pm 34.32$ & $58.85 \pm 18.26$ & $85.42 \pm 24.00$ \\
\hline $\begin{array}{l}\text { Blood pres- } \\
\text { sure medica- } \\
\text { tion }\end{array}$ & 885 & 498 & $145.86 \pm 33.02$ & $59.29 \pm 19.84$ & $96.06 \pm 24.86$ \\
\hline $\begin{array}{l}\text { Cholesterol } \\
\text { Medication }\end{array}$ & 822 & 539 & $146.08 \pm 32.88$ & $59.86 \pm 18.86$ & $96.06 \pm 24.62$ \\
\hline $\begin{array}{l}\text { High Blood } \\
\text { pressure }\end{array}$ & 1100 & 621 & $146.48 \pm 31.49$ & $59.85 \pm 19.64$ & $97.02 \pm 25.67$ \\
\hline $\begin{array}{l}\text { High Choles- } \\
\text { terol }\end{array}$ & 276 & 170 & $143.48 \pm 31.49$ & $58.85 \pm 18.31$ & $94.06 \pm 24.09$ \\
\hline Angina & 83 & 51 & $150.55 \pm 31.04$ & $60.98 \pm 18.75$ & $98.94 \pm 24.77$ \\
\hline Diabetes & 207 & 121 & $144.82 \pm 33.68$ & $60.83 \pm 20.32$ & $97.29 \pm 24.76$ \\
\hline Smoking & 279 & 151 & $145.43 \pm 33.20$ & $61.20 \pm 18.94$ & $93.04 \pm 24.17$ \\
\hline
\end{tabular}
cluded in analysis. LV volumes and mass are reported as mean \pm standard deviation 
Figure 2 shows the first five modes of variation in the functional atlas. Mode 1 shows variation in size at both ED and ES, accounting for $39 \%$ of the variation in the LV morphology of the participants. Mode 2 shows changes in sphericity at both ED and ES which explains $11 \%$ of the variation in LV morphology across the participants. Mode 3 shows a variation in mitral valve tilt at ED similar to that seen in mode 4 of the ED atlas, and variation at wall thickness at ES. Mode 4 shows variation in mitral valve tilt at both ED and ES. Mode 5 can be characterized as variations in apical sphericity at ED and mitral valve tilt at ES.

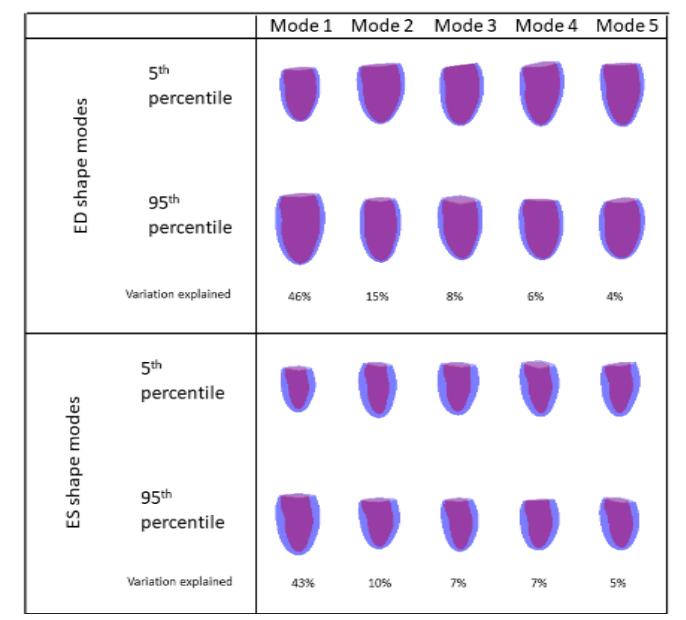

Fig. 1. The first five modes of the ED and ES atlas at the $5^{\text {th }}$ and $95^{\text {th }}$ percentile. The variance explained by each mode is also displayed. Septum is on the left.

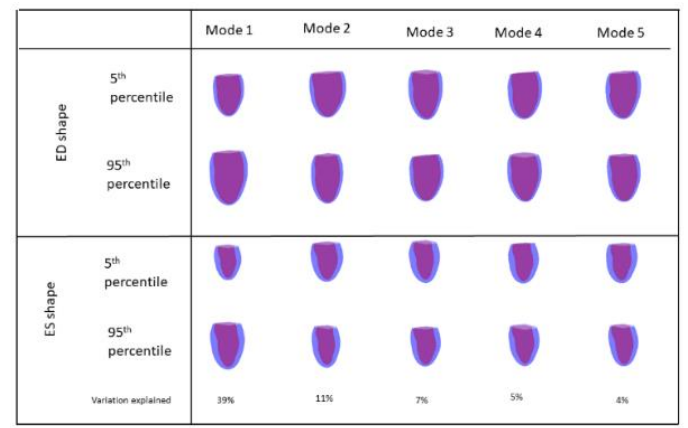

Fig. 2. The first five modes of the function atlas at the $5^{\text {th }}$ and $95^{\text {th }}$ percentile. The variance explained by each mode is also displayed. Septum is on the left.

Figure 3 shows the area under the curve for each risk factor, with each of the four logistic regression models, varying the number of mode scores included. 40 modes were chosen as an appropriate cut off for inclusion in the statistical model as it allowed for the AUC to stabilize in all variables considered. 


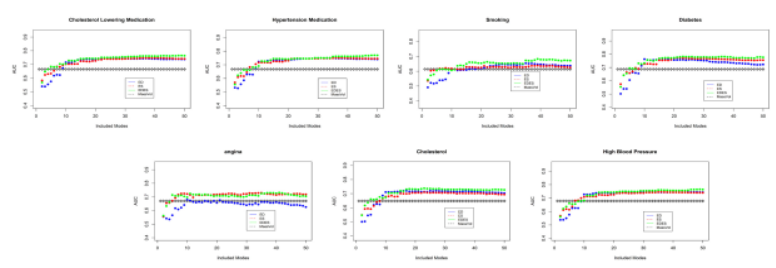

Fig. 3. Cumulative area under the curve plots in a logistic regression model with 1 to 50 modes included. Black is the area under the curve for a mass volume model, blue is ED, red is ES and green is the functional atlas EDES.

The results of the logistic regression models are shown in Table 2 and in Figure 4. Table 2 shows the area under the curve for each model and the result of the Delong's test between the mass volume model and the atlas. The atlases had a significantly higher association with cholesterol lowering medication, blood pressure medication, diabetes, high blood pressure and high cholesterol than LV mass and volume.

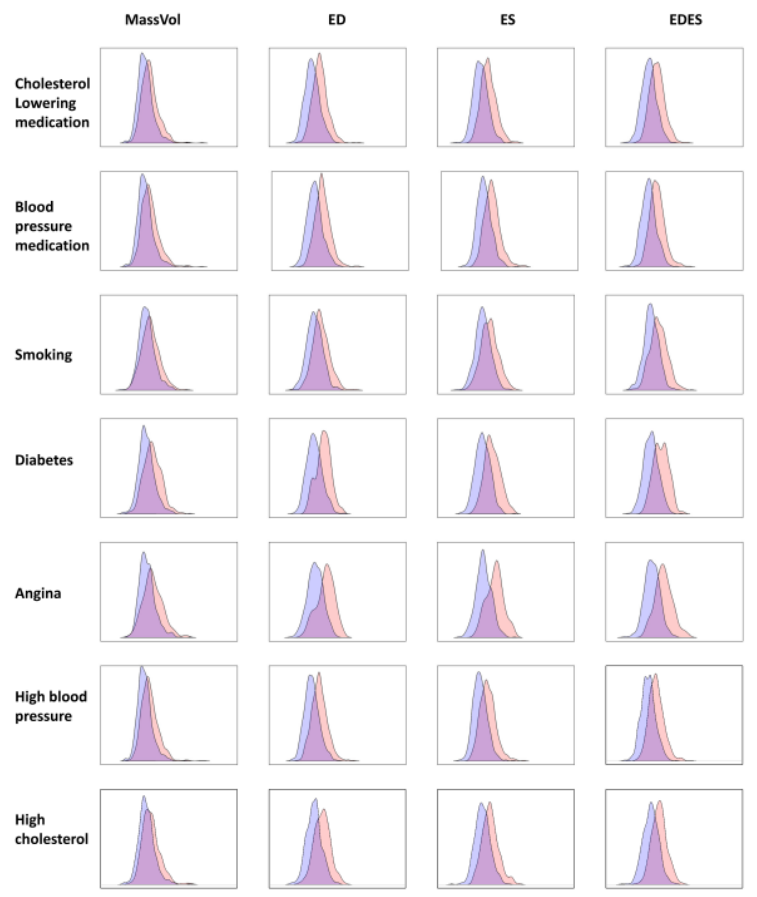

Fig. 4. Density plots of scores from logistic regression models with 40 modes. The risk free cohort are shown in blue and the risk factor positive cases are shown in orange. 
Table 2. Area under the curve for fivefold cross validation logistic regression models for each risk factor. Mass volume model (MV) included LV EDV, ESV and mass, each model built from an atlas used the first 40 modes. * $\mathrm{p}<0.0125$ for Delong's test

\begin{tabular}{lcccc}
\hline & MV & ED & ES & EDES \\
\hline Cholesterol lowering medication & 0.67 & $0.74^{*}$ & $0.75^{*}$ & $0.76^{*}$ \\
Blood pressure medication & 0.67 & $0.74^{*}$ & $0.75^{*}$ & $0.76^{*}$ \\
Smoking & 0.61 & 0.65 & 0.63 & 0.68 \\
Diabetes & 0.69 & 0.73 & $0.76^{*}$ & $0.77^{*}$ \\
Angina & 0.67 & 0.66 & 0.71 & 0.73 \\
High Blood pressure & 0.68 & $0.74^{*}$ & $0.74^{*}$ & $0.76^{*}$ \\
High Cholesterol & 0.65 & $0.72^{*}$ & 0.70 & $0.73^{*}$ \\
\hline
\end{tabular}

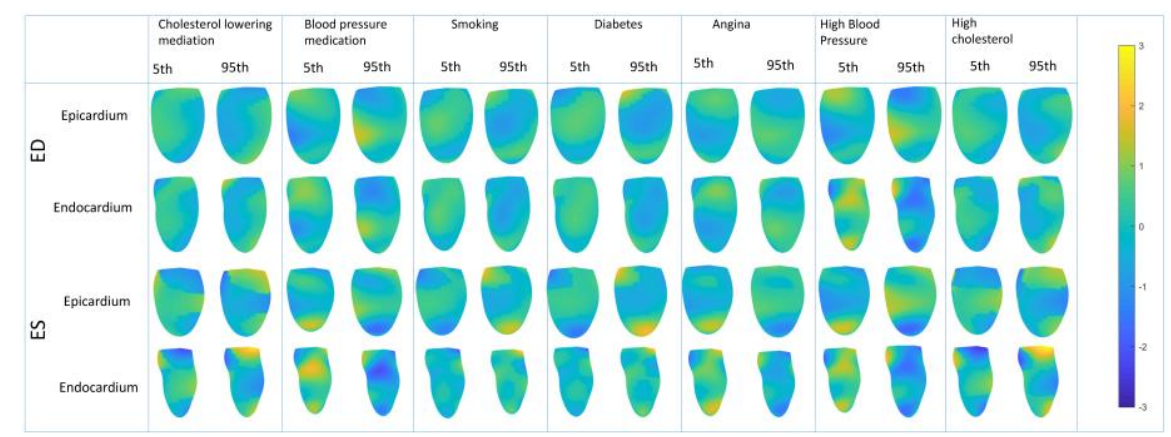

Fig. 5. Morphometric risk factor shapes from the logistic regression model of the functional atlas at the $5^{\text {th }}$ and $95^{\text {th }}$ percentiles. The average shapes were drawn with the color scale shown. Yellow denotes an outward displacement of the surface, and blue an inward displacement. View point is from the anterior with the septum on the left.

\section{Discussion}

The results of this study indicate a strong association between cardiovascular risk factors and LV morphology. Three separate atlases were tested and all showed strong association cholesterol lowering medication, blood pressure medication and high blood pressure. The ED model also showed a strong association with high cholesterol, and the functional atlas (EDES) showed a strong association with diabetes, high blood pressure and high cholesterol. The results suggest that LV morphology changes at both ED and ES in the presence of risk factors. Previously [7] used a five-fold cross-validated logistic regression to test the association of risk factors to two atlases created using different methods. The method used in this paper was similar to the surface atlas in the 
previous work; which built a logistic regression model with the first 20 mode scores at ED and ES combined from independent atlases. Our current study showed similar area under the curves for the risks factors studied, however the ES model and EDES model described in this paper result in a larger area under the curve than ED in most cases. The functional atlas performed similarly to the previous combined score analysis.

The functional atlases was created from the participants' specific shapes at ED and ES, thus having the advantage of describing variation in LV morphology that occur at ED and ES in the same participant. Thus building a logistic regression from this atlas has the advantage of making sure morphological features selected by the logistic regression model at ED and at ES can occur in the same participant.

The study is limited by the cross-sectional nature of UK biobank. However in the future, the shape atlases could be compared with follow-up information, such as cardiac events to better understand remodeling in pre-clinical cardiovascular disease.

\section{Conclusions}

Shape atlases of LV morphology have a stronger relationship with the cardiovascular risk factors of high blood pressure, high cholesterol, and diabetes, than standard measures of mass and volume. The functional atlas has the largest area under the curve, and provides accurate simultaneous deformations at ED and ES.

\section{References}

1. Finegold JA, et al, DPJIjoc (2013) Mortality from ischaemic heart disease by country, region, and age: statistics from World Health Organisation and United Nations 168:934-945

2. Bluemke DA, et al, ARJJotACoC (2008) The relationship of left ventricular mass and geometry to incident cardiovascular events: the MESA (Multi-Ethnic Study of Atherosclerosis) study 52:2148-2155

3. de Marvao A, et al, DPJJCI (2015) Precursors of hypertensive heart phenotype develop in healthy adults: a high-resolution 3D MRI study 8:1260-1269

4. Corden B, et al, DPJJoCMR (2016) Relationship between body composition and left ventricular geometry using three dimensional cardiovascular magnetic resonance 18:32

5. Medrano-Gracia P et al. (2014) Left ventricular shape variation in asymptomatic populations: the multi-ethnic study of atherosclerosis $16: 56$

6. Abdi H, Williams LJJWircs (2010) Principal component analysis 2:433-459

7. Gilbert K et al. (2019) Independent Left Ventricular Morphometric Atlases Show Consistent Relationships with Cardiovascular Risk Factors: A UK Biobank Study Scientific Reports 9:1130 doi:10.1038/s41598-018-37916-6

8. Petersen SE et al. (2017) Reference ranges for cardiac structure and function using cardiovascular magnetic resonance (CMR) in Caucasians from the UK Biobank population cohort 19:18

9. Petersen SE et al. (2015) UK Biobank's cardiovascular magnetic resonance protocol 18:8

10. Abdi H, Williams LJJWircs (2010) Principal component analysis 2:433-459 